Cahiers de la recherche sur les droits fondamentaux

Surveiller et punir / Surveiller ou punir?

\title{
La protection de la santé et de la dignité du détenu par la jurisprudence de la Cour européenne des droits de l'homme
}

Florence Massias

\section{(2) OpenEdition \\ Journals}

Édition électronique

URL : https://journals.openedition.org/crdf/7456

DOI : $10.4000 /$ crdf.7456

ISSN : 2264-1246

Éditeur

Presses universitaires de Caen

Édition imprimée

Date de publication : 1 octobre 2004

Pagination : 23-28

ISBN : 2-84133-221-7

ISSN : $1634-8842$

Référence électronique

Florence Massias, «La protection de la santé et de la dignité du détenu par la jurisprudence de la Cour européenne des droits de l'homme ", Cahiers de la recherche sur les droits fondamentaux [En ligne], 31 2004, mis en ligne le 18 décembre 2020, consulté le 14 novembre 2022. URL : http:// journals.openedition.org/crdf/7456; DOI : https://doi.org/10.4000/crdf.7456 


\title{
La protection de la santé et de la dignité du détenu par la jurisprudence de la Cour européenne des droits de l'homme
}

\author{
Florence MASSIAS \\ Professeur à I'Université de Reims Champagne-Ardenne
}

I. Les exigences générales

A. Quant aux conditions générales de détention

B. Quant aux régimes particuliers de détention

II. La compatibilité de la détention des personnes vulnérables avec l'article 3
A. L'âge et la santé ne sont pas en tant que tels des obstacles à la détention

B. Les conditions de détention des personnes malades ou en fin de vie

La Convention européenne ne comporte aucune garantie spécifique relative à la situation des personnes privées de liberté, a fortiori malades. La Cour européenne vient de nous le rappeler tout récemment avec l'arrêt rendu le 15 janvier 2004: Matencio c. France.

Effectivement, le seul texte intéressant les privations de liberté, l'article 5, offre simplement une protection contre l'arbitraire en garantissant le droit à la sûreté. Il est certainement fondamental, comme verrou de sécurité, pour prémunir les personnes privées de liberté d'atteintes à leurs droits les plus fondamentaux (tortures, disparitions forcées, assassinats), mais il ne concerne pas directement le traitement des personnes privées de liberté.

De ce point de vue, la Convention se distingue du Pacte des Nations Unies sur les droits civils et politiques. L'article 10 du Pacte comporte des dispositions qui concernent spécifiquement le traitement des détenus.

Sur le terrain de la Convention, le détenu pourra se prévaloir de l'ensemble des garanties générales. Dès 1962, la Commission a posé que la détention n'avait pas pour effet de priver des droits garantis par la Convention celui qui a perdu sa liberté. En 1975, c'est la Cour qui, avec son arrêt Golder, a montré que la protection de la Convention s'étend à tous.

Il revient alors aux instances européennes d'adapter ces garanties à la situation particulière des détenus. C'est ainsi par exemple que les détenus peuvent se prévaloir des articles 6, 8, 10 et 12.

Précisons incidemment que le respect de la correspondance du détenu avec la Commission ou la Cour européenne est particulièrement crucial, comme préalable, pour donner effectivité aux droits que lui garantit la Convention, comme vient de le rappeler il y a peu la Cour à propos de l'affaire Cotlet (arrêt du 3 juin 2003), qui a retenu une violation de l'article 8 comme de l'article 34 du fait des obstacles et intimidations relatifs à la correspondance du requérant avec la Commission : ouverture systématique des courriers, retards dans l'acheminement, refus de fournir papier et crayons, mesures d'intimidation des gardiens. Les faits se déroulaient en Roumanie, comme dans l'affaire Petra où la Cour constatait déjà une double violation le 23 juillet 1998. 
Une fois admise l'applicabilité de ces garanties dans le contexte de la détention, la Cour a donc posé que les restrictions aux droits des détenus doivent s'apprécier en fonction « des exigences normales de la détention » (Gol$\operatorname{der} \$ 45)$.

Mais le texte qui fonde la majeure partie des requêtes émanant de détenus, c'est l'article 3 de la Convention qui interdit les traitements inhumains et dégradants, protégeant la dignité du détenu de façon absolue, c'est-à-dire sans restrictions, limitations, dérogations pour quelque raison que ce soit. C'est du reste cette garantie qui était invoquée dans l'affaire Matencio, que nous venons d'évoquer, comme dans plusieurs autres affaires françaises relativement récentes : les affaires Papon (arrêt du 7 juin 2001), Mouisel (arrêt du 14 novembre 2002) et Hénaf (arrêt du 27 novembre 2003).

La majeure partie des revendications des détenus devant la Cour intéressent ainsi leur dignité. Celle-ci serait particulièrement exposée et peu compatible avec la détention.

L'article 10 du Pacte nous le confirme. En effet, nous venons de dire qu'à la différence de la Convention, il garantissait spécifiquement certains droits aux détenus. Le premier étant le droit à la dignité, garanti dès le premier paragraphe de l'article 10: «Toute personne privée de sa liberté est traitée avec humanité et avec le respect inhérent à la personne humaine. » Cette mention particulière du Pacte montre bien les risques que la détention fait courir plus particulièrement à la dignité des personnes privées de liberté. Elle confirme en même temps le caractère absolu du droit.

Le droit à la dignité est absolu : il ne peut en principe composer avec les exigences de la détention (à la différence des droits restrictibles, tels que ceux protégés par l'article 8 par exemple ${ }^{1}$ ). Aucune considération tenant aux nécessités de la détention ne saurait justifier une quelconque atteinte au droit protégé par l'article 3. Pourtant, jusqu'à récemment, peu de requêtes avaient la chance de passer le seuil de la recevabilité. La Commission comme la Cour ne remettaient certes pas en cause le caractère absolu de l'interdit des traitements inhumains et dégradants, mais elles ont rapidement posé que la qualification en tant que telle de traitement inhumain ou de traitement dégradant était relative.

Cette combinaison appréciation relative / droit absolu mérite une brève explication. En pratique, dès lors que la Cour a qualifié le traitement pour le moins de dégradant, l'article 3 s'applique et la protection qu'il offre ne fait l'objet d'aucune limitation. En ce sens le droit est absolu. Mais auparavant, il faut qualifier. Cette qualification du traitement en cause de "dégradant», la détermination du seuil de gravité le plus bas, qui entraîne en même temps l'applicabilité de l'article 3 est relative. De même que la qualification à un échelon supérieur de traitement inhu- main, puis de torture. La détermination du seuil de qualification dépend, comme la Cour le rappelle dans l'arrêt Hénaf, « de l'ensemble des données de la cause, et notamment de la durée du traitement, de ses effets physiques ou mentaux, ainsi que parfois du sexe, de l'âge et de l'état de santé de la victime, donc de sa plus ou moins grande vulnérabilité, et parfois de son propre comportement». Les requêtes des détenus, pour prospérer, doivent donc déjà franchir ce seuil d'applicabilité. Elles se heurtent en outre à des problèmes de preuve, preuve particulièrement difficile à rapporter dans le contexte de la détention.

Qu'il s'agisse de la qualification ou de la preuve, la protection des détenus bénéficie actuellement de l'approche évolutive de la Cour. Approche évolutive quant au seuil de gravité, dont la France a fait deux fois les frais, si l'on peut dire. Avec l'arrêt Selmouni, qui a qualifié de torture des sévices infligés par la police lors d'une garde à vue, sévices qui jusqu’à cette évolution n'auraient été constitutifs que de traitements inhumains. Plus précisément dans notre domaine, avec l'arrêt Hénaf, c'est le seuil d'applicabilité de l'article 3 qui a expressément été abaissé par la Cour.

Ce mouvement vers une élévation des standards concernant l'article 3 était déjà amorcé depuis l'affaire Tomasi (encore une garde à vue en France), avec la reconnaissance de la présomption dite "garde à vue ", concernant la preuve des mauvais traitement infligés par la police. De plus, la Cour retient désormais des violations procédurales de l'article 3, quand les autorités s'abstiennent d'enquêter sur des « allégations défendables » de mauvais traitements de la part de la police ou du personnel pénitentiaire que le requérant ne peut arriver à prouver seul. L'évolution des exigences de la Cour s'est traduite, pour ce qui intéresse le sort des détenus, par une multiplication des décisions de recevabilité et des constats de violation.

Cette jurisprudence évolutive nous conduit à nous intéresser aux décisions les plus récentes de la Cour. Depuis 2000, un corpus jurisprudentiel significatif montre que les détenus peuvent désormais se prévaloir efficacement sur le terrain de l'article 3 d'un droit général à des conditions de détention respectueuses de leur santé et même de leur simple bien-être. Et se pose même, pour les personnes les plus vulnérables, la question de la compatibilité de la détention avec leur handicap, la question de la capacité à la détention.

C'est beaucoup moins efficacement que les détenus pourront invoquer l'article 3 à l'encontre des traitements violents et / ou humiliants du personnel pénitentiaire à leur encontre, et ceci essentiellement pour des questions de preuve (preuve de la réalité des traitements, preuve de leur gravité). Nous ne nous attarderons pas particulièrement sur cet aspect de la protection de la dignité du détenu, mais nous y reviendrons à propos de l'accès aux soins en détention.

1. Une série d'affaires mettent en cause, en 2003 notamment, l'article 8 en même temps que l'article 3, parfois pour les mêmes griefs. Les arrêts Van der Ven et Lorsé rendus contre les Pays-Bas ont constaté une violation de l'article 3 pour des fouilles rectales systématiques au moins une fois par semaine et estimé inutile de se prononcer de ce chef au titre de l'article 8. À titre anecdotique, nous pouvons citer l'affaire Aliev, dans laquelle le grief du requérant concernant l'atteinte à la vie privée que constituerait l'absence de tous rapports conjugaux avec son épouse n'a pu prospérer, malgré une évolution en ce sens dans plusieurs États membres, ce dont la Cour se félicite. 
Nous envisagerons donc d'abord les exigences générales relatives à la compatibilité de la détention avec l'article 3. Puis nous nous attacherons plus particulièrement aux exigences spécifiques relatives à la compatibilité de la détention des personnes vulnérables avec l'article 3 .

\section{Les exigences générales}

Selon la Cour, et comme elle l'a affirmé en 2000 dans l'arrêt Kudla c. Pologne, l'article 3 impose à l'État de s'assurer:

- que tout prisonnier est détenu dans des conditions qui sont compatibles avec le respect de la dignité humaine ;

- que les modalités d'exécution de la mesure ne soumettent pas l'intéressé à une détresse ou à une épreuve d'une intensité qui excède le niveau inévitable de souffrance inhérent à la détention ;

- et que, eu égard aux exigences pratiques de l'emprisonnement, la santé et le bien-être du prisonnier sont assurés de manière adéquate.

Cela signifie d'abord que pèse sur les États une obligation positive d'assurer le respect de la dignité du détenu. Cela veut dire aussi qu'une certaine forme de souffrance et d'humiliation est inhérente à la privation de liberté, et comme telle échappe à la protection de l'article 3. Quant au droit au bien-être et à la santé, dérivés du droit à la dignité, on remarque enfin que la Cour reprend la formule qu'elle emploie pour les droits restrictibles, non absolus : " eu égard aux exigences pratiques de l'emprisonnement». Ce que l'on peut pour le moment en conclure, c'est que la Cour admet que l'emprisonnement atteigne le bienêtre du détenu, et même soit pathogène, mais seulement dans une certaine mesure au-delà de laquelle la dignité sera atteinte et l'article 3 violé.

Qu'il s'agisse du droit au bien-être comme du droit à la santé, c'est encore la question du seuil de gravité qui est posée en même temps que celle de la preuve. Nous nous en tiendrons ici au seuil de gravité ${ }^{2}$.

Ce seuil de gravité peut se trouver atteint du fait des conditions générales de la détention comme du fait de régimes particuliers de détention (quartier de sécurité, isolement, couloir de la mort...).

\section{A. Quant aux conditions générales de détention}

Les affaires concernant les pays de l'Est sont tellement criantes qu'il n'est guère besoin de s'attarder.

L'affaire Kalashnikov (arrêt du 15 juillet 2002) a ainsi conduit à un constat de violation sans état d'âmes : le seuil du dégradant était évidemment dépassé, dès le premier constat de la Cour quant aux faits: une surpopulation carcérale manifeste, le requérant détenu en même temps que 23 autres détenus dans une cellule conçue pour 8 , avec rotation des lits entre 3 détenus. S'ajoutaient l'absence de ventilation des cellules accueillant des fumeurs, le bruit, la lumière allumée en permanence, les conditions de l'usage des toilettes à la vue des codétenus et puis l'infestation par des insectes nuisibles.

Il était inévitable que ces conditions aient des répercussions sur la santé physique (maladies de peau, champignons), comme mentale des détenus.

La violation était évidente pour la Cour « du fait des conditions de détention, en particulier de la surpopulation manifeste, de l'environnement insalubre et de ses effets sur la santé du requérant et sur son bien-être».

La Cour combine tous ces éléments avec la durée pendant laquelle le requérant fut exposé à un tel traitement. Conformément à sa jurisprudence, la Cour refuse de prendre en compte la situation économique difficile des pays concernés.

En 2003, de la même façon, c'est une série d'arrêts concernant l'Ukraine (arrêts du 29 avril 2003) qui constatent des violations de l'article 3. Il ne s'agit plus de suroccupation des cellules, mais à l'inverse d'isolement en quartier de sécurité.

Dans les 6 affaires (Poltoratskiy, Kuznetzov, Khokhlich, Mazarenko, Dankevitch et Aliev), outre les conditions générales de détention, c'est plus particulièrement le régime de détention de sécurité, de détenus mis à l'isolement, qui était en cause.

\section{B. Quant aux régimes particuliers de détention}

Dans toutes ces affaires ukrainiennes, la Cour s'est dit particulièrement préoccupée par le fait que pendant plusieurs mois (plus de trente pour certains), les détenus sont restés enfermés 24 h sur 24 dans leur cellule (individuelle), sans lumière du jour, sans aucune activité de plein air, quasiment sans contact humain. Une détention "dans des conditions inacceptables de ce type constitue déjà un traitement dégradant». Traitement dégradant aggravé par le fait que pendant toute une période, les requérants étaient sous le coup d'une sentence de mort (avant le moratoire de 1997 ayant précédé l'abolition et la commutation en réclusion à perpétuité).

Mais l'isolement en tant que tel n'est pas remis en cause dans ces affaires.

La Cour, dans une affaire Van der Ven (arrêt du 4 février 2003), admet qu'il puisse être justifié pour des raisons de sécurité. Cette même affaire l'a aussi conduite à se prononcer sur les fouilles corporelles.

1) l'isolement: le requérant, soupçonné de préparer une évasion, avait été transféré en quartier de haute sécurité

2. Quant à la preuve, à l'établissement des faits, nous rappelons simplement que la Commission puis la Cour envoient le plus souvent des délégations enquêter sur place; qu'elles tiennent le plus grand compte des rapports du CPT; que les observations de l'OIP et des ONG peuvent aussi jouer un rôle non négligeable, mais qu'ils ne dispensent pas la Cour de rechercher si la personne a personnellement souffert des dysfonctionnements mis à jour. C'est alors que les rapports médicaux prennent leur place. D’une manière générale, le régime de la preuve est assez souple, la Cour n'exige pas une preuve au-delà de tout doute raisonnable, mais admet de simples allégations plausibles dès lors que les autorités ne peuvent pas les réfuter. 
(EBI). Le régime de sécurité se traduisait par une limitation des contacts avec les autres détenus et le personnel de la prison et la réduction des visites aux seuls membres de la famille et à une heure par semaine sans aucun contact physique, sauf une fois par mois: 2 poignées de mains.

Pour la Cour, la détention dans une prison de haute sécurité ne soulève pas en soi de question au titre de l'article 3. Elle relève que les mesures de sécurité étaient très rigoureuses, mais qu'il n'y a pas eu d'isolement sensoriel ou social complet. Dès lors, l'appréciation du seuil de qualification renvoie à l'application pure et simple du principe de proportionnalité. Il s'agit de savoir dans quelle mesure le détenu a été personnellement affecté par le traitement en cause et de savoir si le détenu présentait un risque suffisant pour justifier son placement en quartier de haute sécurité. Eu égard à la gravité des crimes pour lesquels il était poursuivi et au fait qu’il projetait une évasion, la Cour admet l'analyse du risque par les autorités. Elle note par ailleurs que le requérant supportait mal l'isolement et qu'il présentait des syndromes dépressifs. Mais la question des fouilles va éviter à la Cour de prendre clairement parti sur l'isolement.

2) Les fouilles corporelles: le régime de sécurité comprenait des fouilles régulières systématiques. Le requérant $y$ fut soumis chaque semaine pendant trois ans et demi. La Cour se dit ici non convaincue par la nécessité de ces fouilles au regard des exigences de sécurité. En l'absence de nécessités convaincantes en matière de sécurité, les fouilles n'ont pu que susciter chez le détenu des sentiments d'angoisse et d'infériorité susceptibles de l'humilier et de l'avilir. C'est l'absence de nécessité qui permet aux fouilles de routine d'atteindre le seuil de qualification. La Cour conclut que jointes aux autres mesures de sécurité rigoureuses, les fouilles ont constitué un traitement dégradant.

On peut encore évoquer l'affaire Yankov c. Bulgarie (arrêt du 11 décembre 2003) : la Cour a retenu une violation de l'article 3 pour le fait d'avoir rasé le crâne du requérant dans le contexte de son placement en isolement cellulaire ${ }^{3}$, compte tenu de son âge ( 55 ans), et du fait que 9 jours plus tard il avait à comparaître en audience publique. Il est intéressant de rappeler ici la spécificité de ce traitement par rapport aux fouilles à corps ou au port de menottes, spécificité que la Cour, confrontée pour la première fois à ce type de traitement, a tenu à souligner : il s'agit de la modification forcée de l'apparence d'une personne dont celle-ci porte la marque apparente, visible immédiatement par autrui, pendant un certain temps.

Enfin, au vu de l'ensemble de cette jurisprudence récente et quant à l'effet pathogène de la détention, que la dégradation de la santé résulte des conditions matérielles générales ou d'un régime particulier de détention, on remarque que la Cour reste très prudente. Il apparaît que pour elle, les différents troubles, dysfonctionnements, maladies physiques ou mentales imputables directement à la détention ne rendent pas celle-ci pour autant incompatible avec l'article 3. Elle admet un certain degré de pathologie, tant que ces pathologies sont soignées. C'est ce que montre par exemple l'arrêt Khokhlich. Le requérant avait contracté en prison une tuberculose (ce qui apparemment est assez fréquent). Il prétendait avoir été infecté par un codétenu, ce que la Cour estime peu plausible puisqu'il s'agissait de deux formes différentes de tuberculose.

Sans doute, si la contamination avait été plausible, la Cour aurait pu reprocher aux autorités de ne pas avoir rempli leur obligation positive de prévention et de protection du requérant. Mais dès lors que cet élément est écarté, la Cour laisse complètement de côté la question de l'origine de la maladie, pour se contenter du fait que les deux codétenus avaient reçu des soins suffisants et adéquats et qu'ils faisaient l'objet d'une surveillance médicale continue.

Si l'on conçoit que la maladie n'est pas nécessairement incompatible avec l'enfermement, on aimerait au moins que la Cour soit plus claire quant aux obligations positives des États pour limiter le caractère pathogène des prisons.

Qu'elle nous dise à partir de quel seuil de gravité la maladie ne fait plus partie des «souffrances inhérentes » à la privation de liberté.

La maladie n'est donc pas en tant que telle incompatible avec la détention, qu'elle ait été contractée en prison ou non, comme nous allons l'envisager maintenant plus précisément avec la question de la détention des personnes que la maladie ou le grand âge rend particulièrement vulnérables.

\section{La compatibilité de la détention des personnes vulnérables avec l'article 3}

Il s'agit ici plus concrètement de la compatibilité du grand âge et de la maladie avec l'enfermement. Nous disposons ici encore d'un corpus jurisprudentiel important depuis 2001. Nous nous en tenons aux 4 affaires qui ont mis en cause la France : Papon, Mouisel, Hénaf et Matencio: une irrecevabilité, deux constats de violation et un constat de non-violation.

Il résulte de la jurisprudence de la Cour que ni l'âge, ni la santé ne sont en tant que tels incompatibles avec la détention; qu'il appartient seulement aux autorités d'adapter les conditions de détention à la situation particulière des malades et des personnes âgées et de leur offrir un accès à des soins adéquats. Mais, au vu des difficultés que rencontrent les États pour remplir ces obligations positives, la Cour en est venue à admettre que la question de la capacité pénale est aujourd'hui posée face à l'état de santé, l'âge ou un lourd handicap. 


\section{A. L'âge et la santé ne sont pas en tant que tels des obstacles à la détention}

C'est l'enseignement de l'affaire Papon. Celui-ci était âgé de 90 ans au moment où il a déposé sa requête. Il avait fait l'objet d'un triple pontage coronarien deux ans avant sa condamnation. Son cas doit être rapproché de celui de deux autres criminels de guerre (ou contre l'humanité), qui avaient déposé à la même époque le même type de requête, contre l'Italie, pour M. Priebke, et contre le Royaume-Uni, pour M. Sawoniuk. Leurs argumentations sont très proches.

M. Papon prétendait que son âge combiné à son état de santé rendait sa détention contraire à l'article 3. Quant à son âge, et suivant en cela M. Priebke, il disait que l'incarcération d'un homme de plus de 90 ans revenait à une application à peine voilée de la peine de mort, en hâtant la survenue de celle-ci. Quant à sa santé, ce sont surtout les conditions de détention qu'il incriminait.

La Cour observe que dans aucun pays membre du Conseil de l'Europe, l'âge élevé ne constitue en tant que tel un obstacle à la détention. Elle estime toutefois que l'âge, pris en compte avec d'autres facteurs, peut être pris en compte dans certains pays. Elle observe encore qu'aucune disposition de la Convention n'interdit en tant que telle la détention au-delà d'un certain âge. Pour la Cour, ce n'est que dans «certaines conditions que le maintien en détention pour une période prolongée d'une personne d'un âge avancé pourrait poser problème ».

C'est alors qu'il faut avoir égard aux circonstances particulières de l'espèce et c'est ici que la Cour prend en compte l'état de santé du requérant. Les rapports médicaux vont être déterminants. Selon ceux-ci, l'état général est «bon », avec " une conscience et une lucidité parfaites » et le requérant ne montre aucun signe de dépendance. La Cour relève en outre que les conditions de détention sont satisfaisantes. Mais, avant de conclure à l'irrecevabilité, la Cour ouvre une porte:

«Toutefois, si les problèmes de santé du requérant, joints à son grand âge venaient à s'aggraver, la Cour rappelle que le droit français » permet de libérer le requérant. La Cour mentionne ici la possibilité de libération conditionnelle de l'article 729, modifié par la loi du 15 juin 2000, qui mentionne désormais «la nécessité de subir un traitement », et le droit de grâce du président de la République pour raisons humanitaires. À l'époque, la loi du 4 mars 2002 n'était pas encore intervenue pour insérer l'article 720-1-1 dans le CPP dont bénéficiera par la suite M. Papon.

Au moment où la Cour a statué dans l'affaire Mouisel, ce texte était entré en vigueur. Seul l'état de santé de M. Mouisel était en cause. Il avait 48 ans, mais était en fin de vie (leucémie lymphoïde). La Cour commence par réaffirmer ${ }^{4}$ qu'il n'existe pas en tant que telle « une obligation générale de libérer un détenu pour motifs de santé». Mais elle admet «maintenant» que se pose la question de la compatibilité d'un état de santé très préoccupant avec le maintien en détention du requérant dans un tel état. Elle se réfere ici à l'évolution annoncée comme possible par l'arrêt Papon: «Le maintien en détention pour une période prolongée d'une personne d'un âge avancé et de surcroît malade, peut entrer dans le champ de protection de l'article 3. »

Enfin, l'arrêt Mouisel affirme maintenant que «la santé d'une personne privée de liberté fait désormais partie des facteurs à prendre en compte dans les modalités d'exécution de la peine, notamment en ce qui concerne la durée du maintien en détention ». Si la Cour se penche sur les conditions de détention de M. Mouisel, il semble que ce soit presque à titre surabondant.

\section{B. Les conditions de détention des personnes malades ou en fin de vie}

L'affaire Papon correspond au cas de figure de bonnes conditions carcérales générales, de leur adaptation suffisante à son cas particulier et d'un bon suivi médical. L'affaire Mouisel illustre le cas de figure de conditions carcérales générales satisfaisantes, mais de leur inadaptation au cas particulier du requérant et de mauvaises conditions d'accès aux soins. L'affaire Hénaf, pour sa part, mettait exclusivement en cause les conditions d'accès aux soins. Quant à l'affaire Matencio, elle mettait en cause à la fois l'adaptation des conditions de détention et l'accès aux soins.

\section{L'adaptation des conditions de détention à l'état des requérants}

Cet élément est déterminant dans l'irrecevabilité de la requête de $\mathrm{M}$. Papon.

La Cour relève en effet que s'il est certain que le requérant ne bénéficie pas d'une qualité de vie équivalente à celle qu'il aurait s'il était en liberté, les autorités internes ont tenu compte, autant que possible, de son état de santé et de son âge. La plupart des demandes du requérant ont été satisfaites (adaptation de la douche, du vasistas, de la promenade). En revanche, la Cour estime que l'allumage nocturne est inévitable et fait partie des contraintes imposées à tous les détenus, pour des raisons de contrôle et de sécurité.

À l'inverse, l'affaire Mouisel semble montrer que dans certains cas (à un certain seuil de gravité de la maladie), les conditions de détention ne pourront jamais satisfaire au respect de l'article 3 .

La Cour retient un certain nombre d'éléments mis en évidence par les rapports des médecins: la difficulté d'un traitement anticancéreux en maison centrale, l'état psychologique du requérant provoqué par le stress de la maladie et qui a eu des répercussions sur son espérance de vie, la dégradation d'un état de santé de moins en moins conciliable avec la détention. Pour la Cour: «Autant

4. Voir la décision Hatti c. RFA, req. 6181/81, concernant un aveugle et la décision B. c. RFA, req. 13047 / 87, relative à un détenu souffrant de dépression pour avoir passé 5 ans en camp de concentration. Décisions citées par L. Guyon, La Notion juridique de torture en Europe (XII ${ }^{e}$-XX ${ }^{e}$ siècle), Thèse, Université Panthéon-Sorbonne, Paris I, 1994, p. 386. 
d'éléments qui mettent en lumière la progression de la maladie et le caractère difficilement adéquat de la prison pour y faire face, sans que des mesures particulières ne soient prises par les autorités pénitentiaires.» Mais il semble que pour la Cour, ce n'est pas tant l'absence de mesures positives que le simple maintien en détention qui en tant que tel est contraire à l'article 3, pour une personne en fin de vie (qui, la Cour le note quand même, ne constitue plus aucun risque pour la société). Les griefs de M. Mouisel visaient aussi les conditions d'accès aux soins.

\section{L'accès à des soins adéquats}

C'est en fait le comportement du personnel de surveillance qui était en cause dans l'affaire Mouisel, comme dans l'affaire Hénaf.

M. Mouisel, lors de ses transferts en milieu hospitalier, était enchaîné. Ceci n'était pas contesté. M. Mouisel disait qu'il était également menotté et un pied enchaîné au lit pendant les séances de chimiothérapie; il dénonçait aussi la présence des surveillants pendant les soins, ce qui l'avait conduit finalement à les refuser. La Cour ne dispose pas d'éléments pour considérer ces faits comme établis. Pour la Cour, le port des menottes ne pose pas en soi de problème, mais elle considère qu'en l'espèce il était disproportionné, au vu de l'état de santé du requérant, de l'inconfort qui en résultait, de sa faiblesse physique et de l'absence de risque de fuite comme de violence. La violation était de toutes façons déjà acquise.

S’agissant de M. Hénaf, le port d'entrave est le seul point qui fonde la violation, la Cour ayant dans cette affaire décidé d'abaisser le seuil de qualification. Elle commence en effet par rappeler que «la Convention est un instrument vivant à interpréter à la lumière des conditions de vie actuelle ». Elle évoque ensuite son arrêt Selmouni, pour affirmer que « certains actes autrefois exclus du champ d'application de l'article 3 pourraient présenter le degré minimum de gravité requis à l'avenir ».

En l'espèce, compte tenu notamment de l'âge du requérant, de son état de santé, de l'absence d'antécédents faisant sérieusement craindre un risque pour la sécurité, la Cour estime que la mesure d'entrave était disproportionnée au regard des nécessités de la sécurité. L’imposition d'entraves dans ces conditions est contraire à l'article 3.

Malgré cet abaissement du seuil de gravité, la France a échappé à un constat de violation dans l'affaire Matencio (monoplégie d'un membre supérieur, monoparésie d'un membre inférieur, $80 \%$ d'incapacité). Il semble que, comme le regrette la juge Tulkens dans son opinion dissidente, la Cour retienne une lecture très partielle des rapports médicaux.

La Cour relève en l'espèce que, selon un rapport médical le requérant jouissait d'une autonomie qui lui permettait de s'occuper des gestes quotidiens de la vie, de son hygiène, de son alimentation, mais surtout de pouvoir lire et écrire, ce qui paraît pour lui d'une importance capitale. Mais elle omet que le même rapport indiquait que l'état du requérant nécessitait une rééducation fonctionnelle tout à fait pointue irréalisable dans la centrale de Poissy et qu'en outre, les soins qui lui étaient dispensés étaient tout à fait insuffisants. Certes, il semble qu'aucune carence n'ait pu être reprochée aux autorités de Poissy.

En outre, la Cour semble accorder du poids au fait que le requérant ait refusé son transfert à Fresnes. Or, comme le note la juge Tulkens, le requérant n'avait aucune garantie que des soins mieux adaptés lui soient dispensés à Fresnes et de plus, cela l'aurait coupé de son environnement familial. Pour la Cour, la gravité relative de l'état du requérant (absence de dépendance, comme pour M. Papon) et son refus de transfert ont été déterminants dans son constat de non-violation. Il semble aussi que curieusement le rôle de l'OIP dans cette affaire ait joué en défaveur du requérant, la Cour ayant accordé plus de poids à ses constatations (refus de transfert du requérant et soins adéquats à Poissy) qu'aux rapports des médecins pourtant mieux placés pour évaluer la situation du détenu.

Quelles conclusions tirer de ce bref panorama? Quels enseignements?

Peut-être sur le sens et la portée de cette jurisprudence évolutive.

D'abord, il n'est pas anodin que comme dans l'affaire Selmouni, ce soit la France, pays reconnu pour sa tradition démocratique, qui donne l'occasion à la Cour d'élever les standards de protection. Les pays démocratiques doivent en quelque sorte servir de moteur à la promotion des droits de l'homme, sans se laisser freiner par les exemples des violations les plus criantes imputables aux pays nouvellement ralliés au Conseil de l'Europe (la Turquie pour la torture, les pays de l'Est pour le traitement des détenus). S'agissant d'un droit absolu ${ }^{5}$, les États ne peuvent se prévaloir d'aucune marge nationale d'appréciation en arguant de l'écart des pratiques en Europe.

Ensuite, si on peut se féliciter qu'en vertu de cette jurisprudence évolutive la Cour reconnaisse un droit à la santé et au bien-être au détenu sur le terrain de l'article 3, ces droits ne restent que des dérivés du droit à la dignité, ils n'en empruntent pas le caractère absolu.

Enfin, on peut se demander si cette jurisprudence évolutive ira jusqu'à interdire définitivement comme contraires à l'article 3 les peines privatives de liberté, parce que celles-ci sont incapables de remplir leurs objectifs, objectifs que leur assigne notamment l'article $10 \mathrm{du}$ Pacte et que, dès lors qu'elles atteignent nécessairement la santé mentale ou psychique des détenus et cela sans aucune utilité, elles sont dans tous les cas disproportionnées. La question de "la capacité à la détention », que la Cour a récemment posée dans son arrêt Mouisel $^{6}$, ira-telle jusque-là?

\footnotetext{
5. Quant au caractère absolu de ce droit, le caractère relatif de la qualification brouille certes un peu les repères, mais reste en tout état de cause la caractéristique à l'instant évoquée de ne supporter aucune marge nationale d'appréciation.

6. Voir également l'opinion concordante du juge Costa, sous l'arrêt McGlinchey c. Royaume-Uni du 29 avril 2003, concernant les défaillances du suivi à
} l'infirmerie de la prison d'une héroïnomane. 